\title{
Unimolecular fragmentation and radiative cooling of isolated PAH ions: A quantitative study
}

Cite as: J. Chem. Phys. 153, 154303 (2020); https://doi.org/10.1063/5.0027773

Submitted: 31 August 2020 . Accepted: 27 September 2020 . Published Online: 19 October 2020

Mark H. Stockett $\left(\mathbb{D}\right.$, James N. Bull $\left(\mathbb{D}\right.$, Jack T. Buntine, Eduardo Carrascosa $\mathbb{D}^{\mathrm{D}}$, MingChao Ji ${ }^{\mathbb{D}}$, Naoko Kono, Henning T. Schmidt (D), and Henning Zettergren (D)

\section{COLLECTIONS}

Paper published as part of the special topic on 2020 JCP Emerging Investigators Special Collection
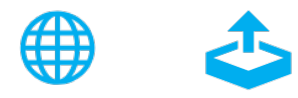

\section{ARTICLES YOU MAY BE INTERESTED IN}

The coverage dependence of the infrared absorption of $\mathrm{CO}$ adsorbed to $\mathrm{NaCl}(100)$

The Journal of Chemical Physics 153, 154703 (2020); https://doi.org/10.1063/5.0025799

Enhanced intersystem crossing of boron dipyrromethene by TEMPO radical

The Journal of Chemical Physics 153, 154201 (2020); https://doi.org/10.1063/5.0025972

Secondary structure specified polarizabilities of residues for an evaluation of circular dichroism spectra of proteins

The Journal of Chemical Physics 153, 155101 (2020); https://doi.org/10.1063/5.0023360

\section{Meet the Next Generation of Quantum Analyzers And Join the Launch

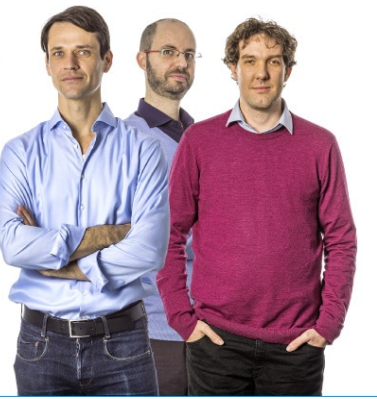

Register now

Zurich

Instruments 


\title{
Unimolecular fragmentation and radiative cooling of isolated PAH ions: A quantitative study
}

\author{
Cite as: J. Chem. Phys. 153, 154303 (2020); doi: 10.1063/5.0027773 \\ Submitted: 31 August 2020 • Accepted: 27 September 2020 • \\ Published Online: 19 October 2020
}

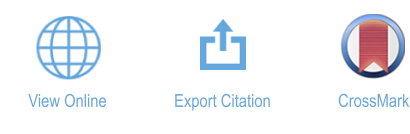

\author{
Mark H. Stockett, ${ }^{1, a)}$ (D) James N. Bull, ${ }^{2}$ (D) Jack T. Buntine, ${ }^{3}$ Eduardo Carrascosa, ${ }^{4}$ (D) MingChao Ji, \\ Naoko Kono,' Henning T. Schmidt,' (D) and Henning Zettergren
}

\begin{abstract}
AFFILIATIONS
${ }^{1}$ Department of Physics, Stockholm University, SE-10691 Stockholm, Sweden

${ }^{2}$ School of Chemistry, University of East Anglia, Norwich Research Park, Norwich NR4 7TJ, United Kingdom

${ }^{3}$ School of Chemistry, University of Melbourne, Parkville, VIC 3010, Australia

"Laboratoire de Chimie Physique Moléculaire, École Polytechnique Fédérale de Lausanne, EPFL SB ISIC LCPM, Station 6, $\mathrm{CH}-1015$ Lausanne, Switzerland
\end{abstract}

Note: This paper is part of the JCP Emerging Investigators Special Collection.

a) Author to whom correspondence should be addressed: mark.stockett@fysik.su.se

\begin{abstract}
Time-resolved spontaneous and laser-induced unimolecular fragmentation of perylene cations $\left(\mathrm{C}_{20} \mathrm{H}_{12}{ }^{+}\right)$has been measured on timescales up to $2 \mathrm{~s}$ in a cryogenic electrostatic ion beam storage ring. We elaborate a quantitative model, which includes fragmentation in competition with radiative cooling via both vibrational and electronic (recurrent fluorescence) de-excitation. Excellent agreement with experimental results is found when sequential fragmentation of daughter ions co-stored with the parent perylene ions is included in the model. Based on the comparison of the model to experiment, we constrain the oscillator strength of the $D_{1} \rightarrow D_{0}$ emissive electronic transition in perylene $\left(f^{R F}=0.055 \pm 0.011\right)$, as well as the absolute absorption cross section of the $D_{5} \leftarrow D_{0}$ excitation transition $\left(\sigma_{a b s}>670 \mathrm{Mb}\right)$. The former transition is responsible for the laser-induced and recurrent fluorescence of perylene, and the latter is the most prominent in the absorption spectrum. The vibrational cooling rate is found to be consistent with the simple harmonic cascade approximation. Quantitative experimental benchmarks of unimolecular processes in polycyclic aromatic hydrocarbon ions like perylene are important for refining astrochemical models.
\end{abstract}

Published under license by AIP Publishing. https://doi.org/10.1063/5.0027773

\section{INTRODUCTION}

Polycyclic Aromatic Hydrocarbons (PAHs) are thought to be ubiquitous in the interstellar medium (ISM) based on the general similarity of their characteristic vibrational frequencies to astronomically observed infrared (IR) emission bands. ${ }^{1,2}$ Numerous astrochemical models have been developed in order to understand the formation and destruction of PAHs and to determine their possible role in, for example, the formation of molecular hydrogen in star-forming regions. ${ }^{3-6}$ These models require accurate physical and chemical data on possible interstellar molecules as well as their precursors and decomposition products. Experimental measurements on key model systems of the proposed astrochemical processes involving PAHs, ${ }^{7}$ such as their destruction by supernova shocks, ${ }^{8}$ are used to refine and calibrate the models.

The archetypical model of PAHs in the ISM considers their destruction by starlight. ${ }^{9}$ In this picture, PAHs absorb ultraviolet (UV) photons with energies exceeding $10 \mathrm{eV}$. This energy is rapidly converted into internal vibrational excitation, initiating a dynamic competition between unimolecular dissociation and radiative cooling. Accurate absolute reaction rates are needed to determine the circumstances under which the excited PAHs will survive long enough to give rise to interstellar emission bands. The purpose of the present report is to quantitatively compare a detailed model of $\mathrm{PAH}$ dissociation and cooling rates to experimental data.

Electrostatic storage devices (ESDs) for $\mathrm{keV}$ ion beams enable highly excited molecular ions to be isolated and probed on 


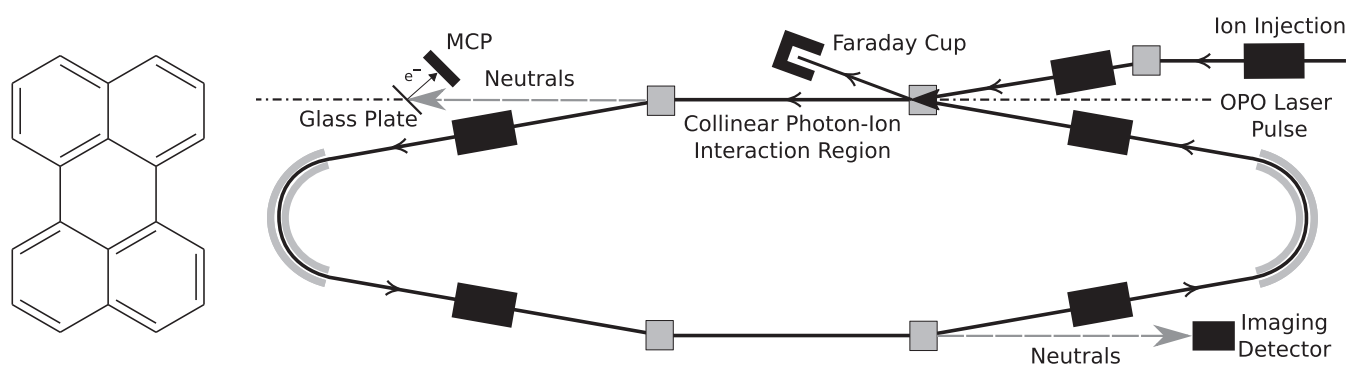

FIG. 1. Left: structure of perylene $\mathrm{C}_{20} \mathrm{H}_{12}{ }^{+}$. Right: schematic of the DESIREE ion storage ring.

timescales exceeding several minutes, allowing quantitative experimental studies on ultra-slow processes, such as vibrational radiative cooling. ESD studies of PAHs ${ }^{10,11}$ and other astrochemically relevant ions ${ }^{12,13}$ have highlighted the important role of recurrent fluorescence (RF) - optical emission from thermally excited electronic excited states ${ }^{14}$-in rapidly reducing their internal energy, saving them from other destruction processes. Very recently, recurrent fluorescence photons emitted by hot naphthalene cations were directly observed in an electrostatic ion beam trap. ${ }^{15}$ Laser probing techniques have been used to investigate the time evolution of the internal energy distribution of highly excited PAHs. ${ }^{16,17}$ Action spectroscopy techniques have provided information on slower, infrared cooling rates ${ }^{18,19}$ as well as photostabilities. ${ }^{20}$

Here, we present a study of the unimolecular fragmentation and radiative cooling of isolated perylene cations $\left(\mathrm{C}_{20} \mathrm{H}_{12}{ }^{+}\right.$, see Fig. 1). Perylene was selected as the subject for this study as there is a considerable wealth of quantitative data available to constrain the model. In particular, perylene is the only $\mathrm{PAH}$ cation for which dispersed fluorescence spectra (in matrix isolation) have been published. ${ }^{21,22}$ Utilizing a cryogenically cooled ESD, we probe the cooling dynamics on timescales 2-3 orders of magnitude slower than previous experiments on $\mathrm{PAH}$ ions performed in room-temperature ESDs.

Two types of experimental data are presented in this work. First, the rate of spontaneous decay of source-heated ions is measured. Second, the stored ion beam is irradiated with laser light resonant with a strong optical excitation of perylene, and the ensuing neutralization yield is measured. These measurements, which are conducted over longer timescales (up to $2 \mathrm{~s}$ ) than previous experiments, are compared to a detailed model that includes unimolecular fragmentation and both electronic and vibrational radiative cooling. Important contributions from the decay of stored daughter ions formed in the sequential $2 \mathrm{H}$-loss reactions $\left(\mathrm{C}_{20} \mathrm{H}_{12}{ }^{+} \rightarrow \mathrm{C}_{20} \mathrm{H}_{11}{ }^{+}+\mathrm{H}\right.$ $\rightarrow \mathrm{C}_{20} \mathrm{H}_{10}{ }^{+}+2 \mathrm{H}$ ) are also included in the model. By adjusting the model parameters to fit the experimental results, we obtain quantitative information on the transition probabilities for two electronic transitions of $\mathrm{C}_{20} \mathrm{H}_{12}{ }^{+}$.

\section{EXPERIMENTS}

Experiments were conducted at the DESIREE (Double ElectroStatic Ion Ring ExpEriment) infrastructure at Stockholm University. ${ }^{23}$ Cryogenic cooling of the DESIREE storage ring, which is schematically shown in Fig. 1 , to $\approx 13 \mathrm{~K}$ results in a residual gas density on the order of $\sim 10^{4} \mathrm{~cm}^{-3} \cdot{ }^{24}$ Using cryogenic ESDs, ions may be stored in an essentially collision-free environment for times exceeding hundreds of seconds, ${ }^{25}$ enabling investigations of radiative cooling dynamics on ultra-slow timescales, i.e., seconds to minutes. $^{26-30}$

Perylene vapor was sublimed from powder in a resistively heated oven coupled to an electron cyclotron resonance (ECR) ion source (Pantechnik Monogan). Cations extracted from the source were accelerated to $15 \mathrm{keV}$, and $\mathrm{C}_{20} \mathrm{H}_{12}{ }^{+}(\mathrm{m} / z=252)$ was selected with a bending magnet. Mass spectra, obtained by scanning the magnetic field, showed extensive fragmentation of the perylene ions, with losses of up to $8 \mathrm{H}$ atoms. The high degree of fragmentation suggests that the ions are produced with a broad distribution of internal energy. Mass-selected beams of $\mathrm{C}_{20} \mathrm{H}_{12}{ }^{+}$were stored in the DESIREE ion storage ring illustrated in Fig. 1.

Immediately after ion injection into the DESIREE storage ring, neutral fragments are formed from $\mathrm{C}_{20} \mathrm{H}_{12}{ }^{+}$ions, which still retain significant internal energy from their time of formation in the ion source. Neutrals formed in one of the two straight sections of the storage ring continue with high velocity toward microchannel plate (MCP) detectors. The measured neutral yield per revolution around the ring as a function of time $t$ after the ions left the source is $R(t)$. At the end of each storage cycle, the number of ions remaining in the ring $N_{\text {stored }}$ is measured by dumping the beam into a Faraday cup. The MCP detector background is measured in the brief interval between injections. By recording $N_{\text {stored }}$ while varying the cycle time, the $1 / \mathrm{e}$ beam storage lifetime was measured as $\tau_{\text {store }}=290 \pm 20 \mathrm{~s}$. The storage lifetime is assumed to be limited by collisions with residual gas.

For the experiments presented in this work, only the "Glass Plate" detector on the ion injection side of DESIREE was used, as it utilizes custom ultra-high dynamic range MCPs (Photonis), which are suitable for high count rates at cryogenic temperatures. ${ }^{31} \mathrm{Neu}$ trals striking this detector impact a glass plate with a thin gold coating, which emits secondary electrons that are detected by the MCP. This arrangement allows for colinear overlap of laser light with the ion beam in this straight section.

An optical parametric oscillator (OPO) laser system (EKSPLA $\mathrm{NT} 242,1 \mathrm{kHz}$ frequency) provided light at wavelengths resonant with the electronic transitions of $\mathrm{C}_{20} \mathrm{H}_{12}{ }^{+}(535 \mathrm{~nm})$. An example of the laser-induced neutral yield is shown in Fig. 2. Photo-excited ions may continue to circulate in the storage ring for several milliseconds before succumbing to unimolecular fragmentation, leading to 


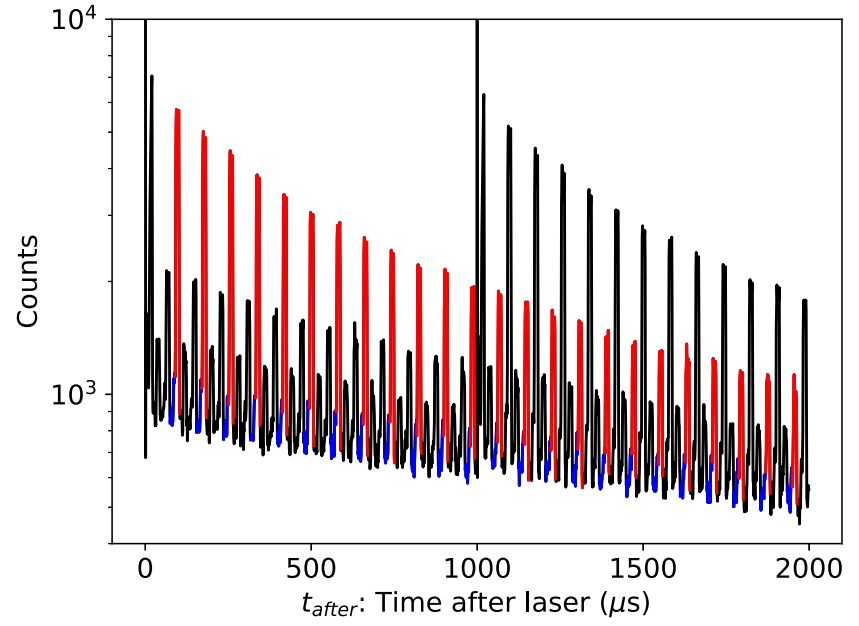

FIG. 2. Example of the detector signal during the laser excitation experiment. Signal due to the laser light striking the detector is seen at $t_{\text {after }}=0 \mathrm{~ms}$ and $1 \mathrm{~ms}$ (peaks off scale). The peaks in red are the laser-induced neutral yield due to the laser pulse at $t_{\text {after }}=0 \mathrm{~ms}$. The peaks are separated by the revolution time of the laser-excited segment of the stored ion beam $(80.9 \mu \mathrm{s})$. The blue areas just before each red peak are subtracted as background. The remaining black peaks are series of neutral fragments due to other laser shots.

a periodic series of neutral signal peaks each time the ions return to the straight section. Each peak is integrated, and the counts in a background window prior to each laser-induced peak are subtracted. This gives the laser-induced count rate $R_{e}$ as a function of both laserfiring time $t_{\text {laser }}$ and the time after the laser firing time $t_{\text {after }}$ at which the neutrals were formed.

Note that in several figures in this work, experimental data are plotted on a logarithmic time scale. For these plots, the data have been binned with a bin width that increases with time, yielding evenly spaced points.

\section{MODELING}

\section{A. Rate constants}

The spontaneous and laser-induced fragmentation rates are modeled using a statistical theory. The model starts from the vibrational density of states $\rho$ computed with the Beyer-Swinehart algorithm $^{32}$ and harmonic oscillator vibrational mode frequencies $v_{s}$ calculated at the $\omega$ B97X-D//aug-cc-pVTZ level of Density Functional Theory (DFT) in Gaussian 16. ${ }^{33-35}$ Rotations are neglected. Separate DFT calculations were performed for the parent $\mathrm{C}_{20} \mathrm{H}_{12}{ }^{+}$and $\mathrm{C}_{20} \mathrm{H}_{11}{ }^{+}$daughter ions. The calculated vibrational frequencies are tabulated in the supplementary material.

Infrared (IR) radiative cooling rate constants were calculated within the Simple Harmonic Cascade (SHC) approximation. ${ }^{29}$ For a vibrational total energy $E$, the infrared radiative cooling rate coefficient $k_{s}^{I R}$ for a given mode $s$ is ${ }^{12}$

$$
k_{s}^{I R}(E)=A_{s}^{I R} \sum_{v=1}^{v \leq E / h v_{s}} \frac{\rho\left(E-v h v_{s}\right)}{\rho(E)},
$$

where $h$ is Planck's constant. In the SHC model, only transitions that involve the vibrational quantum number $v$ decreasing by one quantum are considered, i.e., $\Delta v=-1$. The Einstein coefficients $A_{s}^{I R}$ for $v=1 \rightarrow v=0$ transitions were taken from our DFT calculations (see the supplementary material). The total IR cooling rate constant is given by $k_{t o t}^{I R}=\sum_{s} k_{s}^{I R}$. Figure 3 shows the rate constants for the five brightest modes of $\mathrm{C}_{20} \mathrm{H}_{12}{ }^{+}$, which account for about $80 \%$ of the total rate constant $k_{\text {tot }}^{I R}$. All $40 \mathrm{IR}$ active modes of $\mathrm{C}_{20} \mathrm{H}_{12}{ }^{+}$are included in the simulation.

For the $\mathrm{C}_{20} \mathrm{H}_{11}{ }^{+}$daughter ion, the calculated lowest-energy structure is slightly non-planar. The lack of planar symmetry leads to reduced activity of the brightest modes and non-zero activity for all 90 modes. The total vibrational emission rate constant for $\mathrm{C}_{20} \mathrm{H}_{11}{ }^{+}$ is $\hat{k}_{t o t}^{I R}$, with the hat indicating a rate constant for $\mathrm{C}_{20} \mathrm{H}_{11}{ }^{+}$.

The rate constant for Recurrent Fluorescence (RF), i.e., emission from a thermally populated electronic excited state, is calculated using the following expression: ${ }^{36}$

$$
k_{j}^{R F}(E)=\tilde{A}_{j}^{R F} \frac{\rho\left(E-h v_{j}\right)}{\rho(E)},
$$

where the transition energies $h v_{j}$ are taken from the fluorescence spectrum in matrix isolation reported by Chillier et al. ${ }^{22}$ Experimental values for the Einstein coefficients are unavailable so they are assumed to be proportional to the experimental relative intensities $f_{j}$, i.e., $\tilde{A}_{j}^{R F}=\frac{2}{3} f_{j}\left(h v_{j}\right)^{2}$. The tilde over $A$ in Eq. (2) indicates that these are relative values, which must be scaled to some absolute transition rate. The vibronic transitions included in the model, which dominate the fluorescence spectrum are given in Table I. All of these transitions are assigned to the $D_{1} \rightarrow D_{0}$ emissive electronic transition. ${ }^{22}$ The total RF cooling rate is $k_{t o t}^{R F}=f^{R F} \sum_{j} k_{j}^{R F}$, where $f^{R F}$ is taken as a free parameter, and corresponds to the emission oscillator strength, which provides the absolute scaling. The RF rate constants are plotted in Fig. 4, assuming $f^{R F}=0.05$.

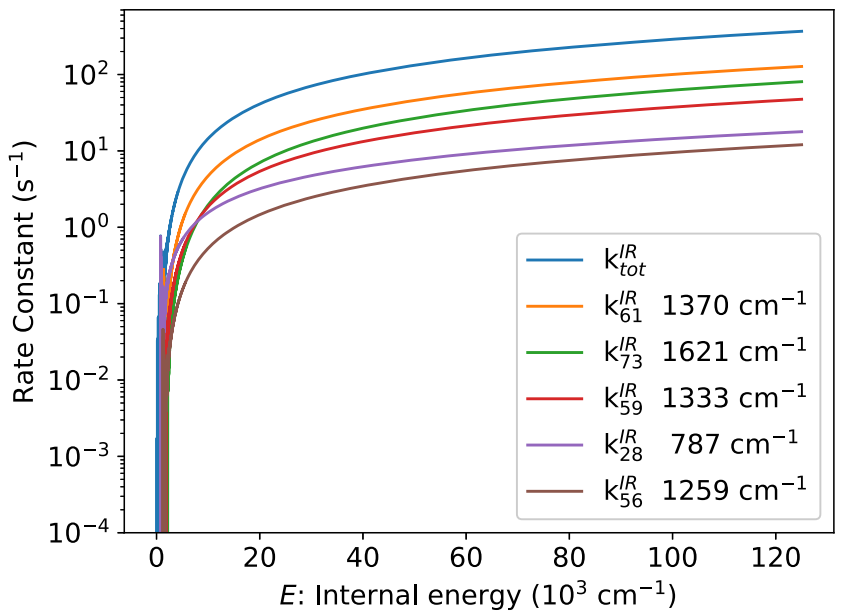

FIG. 3. Infrared radiative cooling rate constants [Eq. (1)] for the five brightest vibrational modes of $\mathrm{C}_{20} \mathrm{H}_{12}{ }^{+}$, and the total rate constant $k_{\text {tot }}^{I R}$. The irregular behavior close to $E=0$ is due to unevenness in the density of states at these energies. See the supplementary material for a tabulation of all vibrational modes. 
TABLE I. Energies and weights from Ref. 22 of electronic transitions included in the recurrent fluorescence rate model. The relative Einstein coefficients $\tilde{A}_{j}^{R F}$ are scaled by the oscillator strength $f^{R F}$ to calculate the absolute emission rates.

\begin{tabular}{lccc}
\hline \hline$j$ & $h v_{j}\left(\mathrm{~cm}^{-1}\right)$ & $f_{j}$ & $\tilde{A}_{j}^{R F}\left(10^{7} \mathrm{~s}^{-1}\right) \mathrm{r}$ \\
\hline 1 & 12623 & 0.24 & 2.5 \\
2 & 12266 & 0.17 & 1.8 \\
3 & 11325 & 0.26 & 2.3 \\
4 & 11236 & 0.11 & 1.0 \\
5 & 11026 & 0.21 & 1.7 \\
Tot. & & 1.00 & 9.2 \\
\hline
\end{tabular}

RF is not included in the model for the $\mathrm{C}_{20} \mathrm{H}_{11}{ }^{+}$daughter ion due to the lack of experimental data on its electronic transitions. Furthermore, the RF rate constant would need to be implausibly high-two orders of magnitude greater than that for $\mathrm{C}_{20} \mathrm{H}_{12}{ }^{+}-$to be competitive with dissociation (see below).

Unimolecular fragmentation rate constants were based on those derived by West et al., ${ }^{37}$ who used the Rice-RamspergerKassel-Marcus (RRKM) theory to fit breakdown curves for PAHs following vacuum ultraviolet photon absorption. Three reaction channels were modeled by West et al. (see Table II), $\mathrm{H}$ - and $\mathrm{H}_{2}$ loss from $\mathrm{C}_{20} \mathrm{H}_{12}{ }^{+}$, and the sequential $2 \mathrm{H}$-loss reaction $\mathrm{C}_{20} \mathrm{H}_{12}{ }^{+} \rightarrow$ $\mathrm{C}_{20} \mathrm{H}_{11}{ }^{+}+\mathrm{H} \rightarrow \mathrm{C}_{20} \mathrm{H}_{10}{ }^{+}+2 \mathrm{H}$. For consistency with the present model, we have recalculated the dissociation rate constants using our calculated vibrational frequencies in terms of the inverse Laplace transform approximation,

$$
k_{R}^{\text {diss }}(E)=A_{R}^{\text {diss }} \frac{\rho\left(E-E_{R}^{\text {diss }}\right)}{\rho(E)},
$$

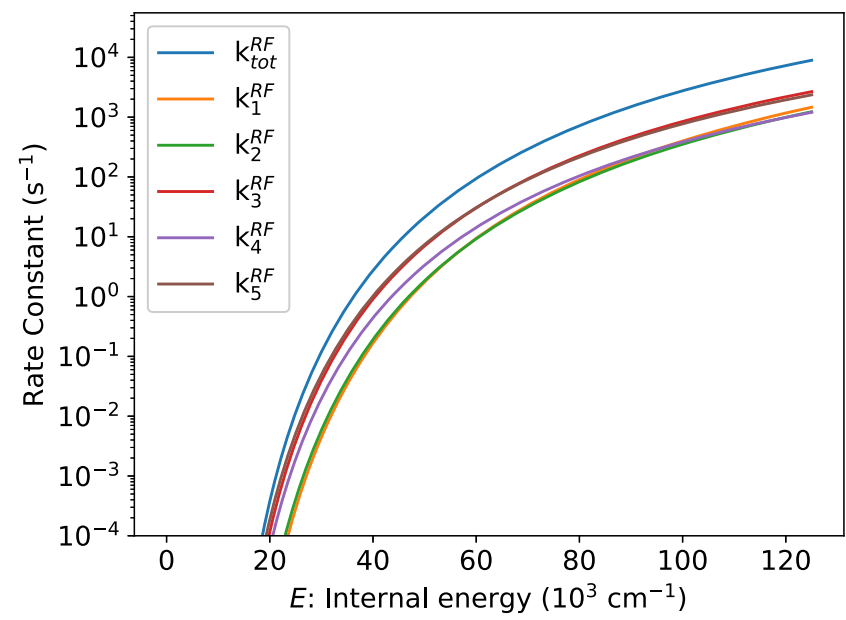

FIG. 4. Recurrent fluorescence radiative cooling rate constants [Eq. (2)] for emissive electronic transitions of $\mathrm{C}_{20} \mathrm{H}_{12}{ }^{+}$in Table I and the total rate constant $k_{\text {tot }}^{R F}$.
TABLE II. Unimolecular fragmentation channels included in the model. Dissociation energies $E_{R}^{\text {diss }}$ are taken from West et al., ${ }^{37}$ and the pre-exponential factors $A_{R}^{\text {diss }}$ are chosen to match their tabulated rates.

\begin{tabular}{lccc}
\hline \hline$R$ & Reaction & $E_{R}^{\text {diss }}(\mathrm{eV})$ & $A_{R}^{\text {diss }}\left(\mathrm{s}^{-1}\right)$ \\
\hline $\mathrm{H}$ & $\mathrm{C}_{20} \mathrm{H}_{12}{ }^{+} \rightarrow \mathrm{C}_{20} \mathrm{H}_{11}{ }^{+}+\mathrm{H}$ & 4.75 & $3.0 \times 10^{16}$ \\
$\mathrm{H}_{2}$ & $\mathrm{C}_{20} \mathrm{H}_{12}{ }^{+} \rightarrow \mathrm{C}_{20} \mathrm{H}_{10}{ }^{+}+\mathrm{H}_{2}$ & 4.58 & $6.2 \times 10^{15}$ \\
$2 \mathrm{H}$ & $\mathrm{C}_{20} \mathrm{H}_{11}{ }^{+} \rightarrow \mathrm{C}_{20} \mathrm{H}_{10}{ }^{+}+\mathrm{H}$ & 2.63 & $1.5 \times 10^{13}$ \\
\hline \hline
\end{tabular}

where the dissociation energies $E_{R}^{\text {diss }}$ were taken from West et al., and the pre-exponential factors $A_{R}^{\text {diss }}$ were chosen to fit their tabulated rate constants. These values are given in Table II. The total fragmentation rate constant for $\mathrm{C}_{20} \mathrm{H}_{12}{ }^{+}$is the sum of the rate constants for $\mathrm{H}-$ and $\mathrm{H}_{2}$-loss channels and the rate for destruction through collisions with residual gas, i.e., $k_{\text {tot }}^{\text {diss }}=k_{\mathrm{H}}^{\text {diss }}+k_{\mathrm{H}_{2}}^{\text {diss }}+1 / \tau_{\text {store }}$. The rate constant for sequential $2 \mathrm{H}$-loss $\hat{k}_{2 \mathrm{H}}^{\text {diss }}$, which affects only the $\mathrm{C}_{20} \mathrm{H}_{11}{ }^{+}$ daughter ions, is calculated using the corresponding ion's density of states. No further adjustments are made to the dissociation rate constants.

Figure 5 shows all the rate constants included in the simulation, again assuming $f^{R F}=0.05$. These rate constants are tabulated in the supplementary material.

\section{B. Master equation simulation}

The time evolution of the population distribution $g(E, t)$ of intact perylene cations was simulated using the master equation approach. ${ }^{39}$ Starting from an initial Boltzmann distribution of vibrational energy $g(E, t=0)$ normalized such that $N(0)=\int g(E, t=0) d E$ $=1$, radiative cooling propagates the distribution according to the master equation,

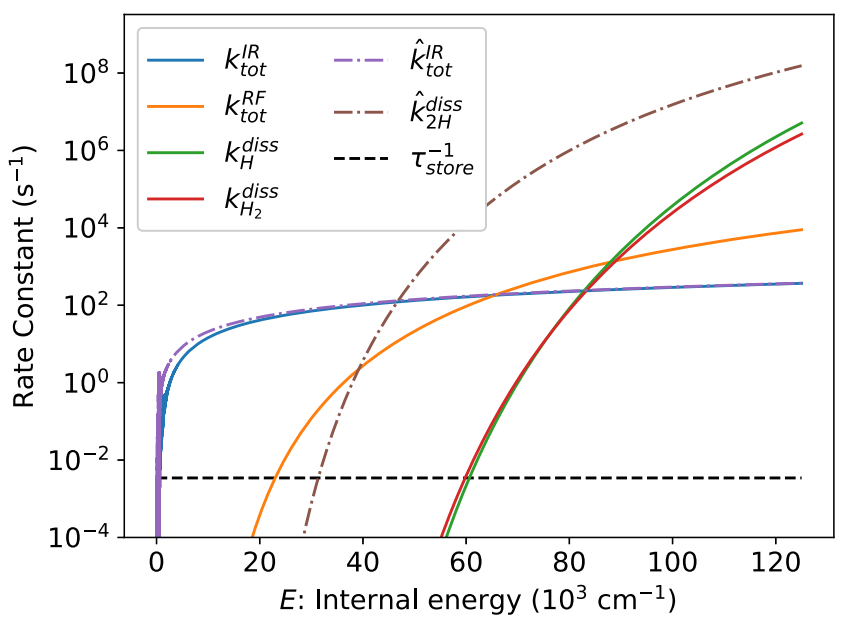

FIG. 5. Rate constants included in master equation fragmentation and radiative cooling simulations for $\mathrm{C}_{20} \mathrm{H}_{12}{ }^{+}$(solid lines). Dashed-dotted lines indicate rate constants for the $\mathrm{C}_{20} \mathrm{H}_{11}{ }^{+}$daughter ions. The beam storage lifetime is $\tau_{\text {store }}$. 


$$
\frac{d}{d t} g\left(E_{i}, t\right)=\sum_{j \neq i}\left[k_{i \leftarrow j} g\left(E_{j}, t\right)-k_{i \rightarrow j} g\left(E_{i}, t\right)\right] .
$$

The matrix elements $k_{i \leftarrow j}$ and $k_{i \rightarrow j}$ are the radiative cooling rate constants connecting energy levels $i$ and $j$, e.g., $k_{i \rightarrow j}=k_{s}^{I R}\left(E_{i}\right)$, where $E_{i}-E_{j}=h v_{s}$. A similar construction applies to the RF rate constants. Fragmentation depletes the population by $d g\left(E_{i}, t\right) / d t$ $=-k_{\text {tot }}^{\text {diss }}\left(E_{i}\right) g\left(E_{i}, t\right)$. The simulation time step $d t$ is set dynamically in inverse proportion to the highest rate constant at the energy $E_{\max }$ of the high-energy edge of $g(E, t)$, here taken to be the energy where the distribution is most rapidly changing in the simulation [where $g(E, t)-g(E, t+d t)$ was maximized in the previous step].

Formation and eventual decay of the ionic products of the reactions in Table II are accounted for in the simulation. In particular, the $\mathrm{C}_{20} \mathrm{H}_{11}{ }^{+}$daughter ions formed in the $\mathrm{H}$-loss reaction are assumed to be stored with some probability $\epsilon_{\text {store }}$, and their population distribution $\hat{g}(E, t)$ evolves according to the IR cooling and dissociation rates of that system, $\hat{k}_{\text {tot }}^{I R}$ and $\hat{k}_{2 H}^{\text {diss }}$.

The rate of fragmentation $\Gamma(t)$ is equal to the rate of change of the number of intact ions $N(t)=\int g(E, t) d E$, i.e.,

$$
\Gamma(t)=-\frac{d N(t)}{d t}=\int k_{\text {tot }}^{\text {diss }}(E) g(E, t) d E .
$$

For an internal energy distribution narrow enough to be approximated as $g(E, t) \propto \delta(E)$, Eq. (5) returns a single exponential decay curve. However, for broad internal energy distributions, such as those produced by the ECR ion source used here, and given that the dissociation rate constants depend strongly on the internal energy, the fragmentation rate is better approximated as a power law $\Gamma(t)=\Gamma_{0} t^{-P}(P \approx 1) .{ }^{40,41}$ Hansen et al. derived an approximate value (neglecting radiative effects) for the prefactor, ${ }^{41}$

$$
\Gamma_{0}=g\left(E_{m}\right) / \frac{d\left(\ln \left(k_{\text {tot }}^{\text {diss }}\left(E_{m}\right)\right)\right.}{d t},
$$

where $E_{m}(t)$ (not to be confused with $E_{\max }$ ) is the internal energy defined by $k_{\text {tot }}^{\text {diss }}\left(E_{m}\right)=1 / t$.

Inclusion of radiative cooling modifies the simulated decay rate by shifting population density toward lower energy. After a critical time $\tau_{c}$, the radiative decay rate dominates over dissociation and the fragment yield drops, giving a so-called quenched power law,

$$
\Gamma(t)=\Gamma_{0} t^{-P} e^{-t / \tau_{c}}
$$

Eq. (7) is used as a fitting function to analyze both the experimental and simulated yield curves.

\section{RESULTS AND DISCUSSION}

\section{A. Spontaneous decay}

\section{Spontaneous neutral yield}

The measured rate of neutral fragment detection $R(t)$ is shown in Fig. 6 (right vertical axis). The experimental rate (measured as

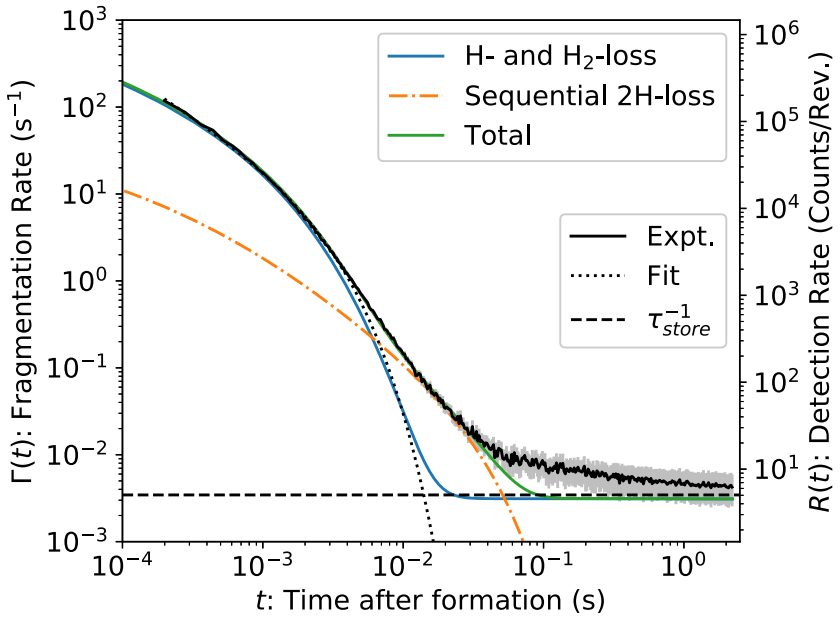

FIG. 6. Spontaneous neutral yield $\Gamma(t)$ from stored beam of source-heated $\mathrm{C}_{20} \mathrm{H}_{12}{ }^{+}$ (left vertical axis). The right vertical axis is the raw experimental count rate $R(t)$, related to $\Gamma(t)$ by Eq. (8). The black line is the experimental measurement, with the gray area giving the uncertainty due to counting statistics. The black dotted line is a fit of the experimental data to Eq. (7). The beam storage lifetime is $\tau_{\text {store }}$. The colored lines are the results of our master equation simulations (assuming $T_{0}=$ $1800 \mathrm{~K}, f^{R F}=0.05$, and $\epsilon_{\text {store }}=25 \%$ ). The simulated yield includes contributions from fragmentation of $\mathrm{C}_{20} \mathrm{H}_{12}{ }^{+}$by $\mathrm{H}$ - and $\mathrm{H}_{2}$-loss (solid line) and the subsequent fragmentation of the $\mathrm{C}_{20} \mathrm{H}_{11}{ }^{+}$daughter ions through sequential $2 \mathrm{H}$-loss (dasheddotted line). Vibrational and electronic radiative cooling are also included in the simulation.

particles per revolution) is related to the actual per-particle fragmentation rate by $^{24}$

$$
R(t)=\frac{\epsilon_{\text {det }} L N_{\text {stored }}}{f C} \Gamma(t),
$$

where $N_{\text {stored }}$ is the number of ions stored (measured at the end of each cycle), $\epsilon_{\text {det }}$ is the detector efficiency, $L=0.95 \mathrm{~m}$ is the length of the straight section seen by the detector, $f=12.35 \mathrm{kHz}$ is the revolution frequency ( $80.9 \mu$ s revolution period), and $C=8.7 \mathrm{~m}$ is the circumference of the storage ring. Of these parameters, only $\epsilon_{\text {det }}$ is poorly known. It is determined here by requiring that the derived fragmentation rate be always greater than the collisional destruction rate implied by the beam storage lifetime, i.e., $\Gamma>\tau_{\text {store }}^{-1}$. This suggests a detector efficiency $\epsilon_{\text {det }} \approx 2 \%$, which is consistent with absolute detection efficiencies of MCPs for low-energy atoms. ${ }^{42,43}$ An $\mathrm{H}$ atom fragment $(1 \mathrm{Da})$ exiting the ring with the same velocity as its parent ion $(252 \mathrm{Da})$ carries $1 / 252$ of the $15 \mathrm{keV}$ kinetic energy, or $60 \mathrm{eV}$. The resulting absolute values of $\Gamma(t)$ are given on the left vertical axis of Fig. 6.

A fit to Eq. (7) with $P=1$ (fixed) gives $\tau_{c}=2.22 \pm 0.01 \mathrm{~ms}$ and represents the decay curve well for the first $5 \mathrm{~ms}$, after which the fit strongly diverges (dotted line in Fig. 6). The fitted value of $\Gamma_{0}=2.71( \pm 0.01) \times 10^{-2} \mathrm{~s}^{-1}$ is similar to the approximate value [Eq. (6)] of $1.0 \times 10^{-2} \mathrm{~s}^{-1}$ reported by the simulation at times close to the first experimental data point, where radiative effects not included in the approximation leading to Eq. (6) are minimal. This further supports our absolute scaling of the decay rate. 
Figure 6 also includes the results of our master equation simulations assuming an initial temperature of $T_{0}=1800 \mathrm{~K}$. Fragmentation of $\mathrm{C}_{20} \mathrm{H}_{12}{ }^{+}$by $\mathrm{H}$ - and $\mathrm{H}_{2}$-loss gives a neutral yield rate, which closely follows a quenched power law [Eq. (7)] and agrees with the experimental data for the first $5 \mathrm{~ms}$. Between $5 \mathrm{~ms}$ and $50 \mathrm{~ms}$, there is a large underestimation by theory compared with the experimental rate, reaching an order of magnitude at $\approx 20 \mathrm{~ms}$. It is possible to reproduce the shape of the experimental decay curve at these times by including the sequential decay of the $\mathrm{C}_{20} \mathrm{H}_{11}{ }^{+}$daughter ion by sequential $2 \mathrm{H}$-loss. The simulation in Fig. 6 assumes that $\epsilon_{\text {store }}=$ $25 \%$ of the $\mathrm{C}_{20} \mathrm{H}_{11}{ }^{+}$daughter ions are stored in the ring. The remaining difference between the simulated and experimental decay rates at times longer than $50 \mathrm{~ms}$ may be due to decay channels not included in the simulation, such further fragmentation of the $\mathrm{C}_{20} \mathrm{H}_{10}{ }^{+}$daughter ions. Recall that losses of up to $8 \mathrm{H}$ atoms were observed in the ion source mass spectrum.

In addition to the initial temperature and the storage probability, the only other parameter adjusted to give the decay curves in Fig. 6 is the recurrent fluorescence oscillator strength $f^{R F}=0.05$. Published quantum chemical calculations ${ }^{44-48}$ provide oscillator strengths for the lowest optical transition of perylene cations ranging from $10^{-4}$ to $10^{-2}$ (note that we assume absorption and emission oscillator strengths are equal as supported by the measured ${ }^{22}$ Stokes shift $<50 \mathrm{~cm}^{-1}$ ). Our value is somewhat beyond the high end of this range and may serve as a benchmark for future calculations, or for different models of the RF process itself. For example, other studies have included contributions to the RF rate due to emission from vibronically excited states. ${ }^{11,36}$ These contributions, however, amounted to only a $10 \%$ increase in the total RF rate constant.

\section{Sensitivity to model parameters}

Figure 7 shows several runs of the master equation simulation with different values of $f^{R F}$ while keeping the initial temperature

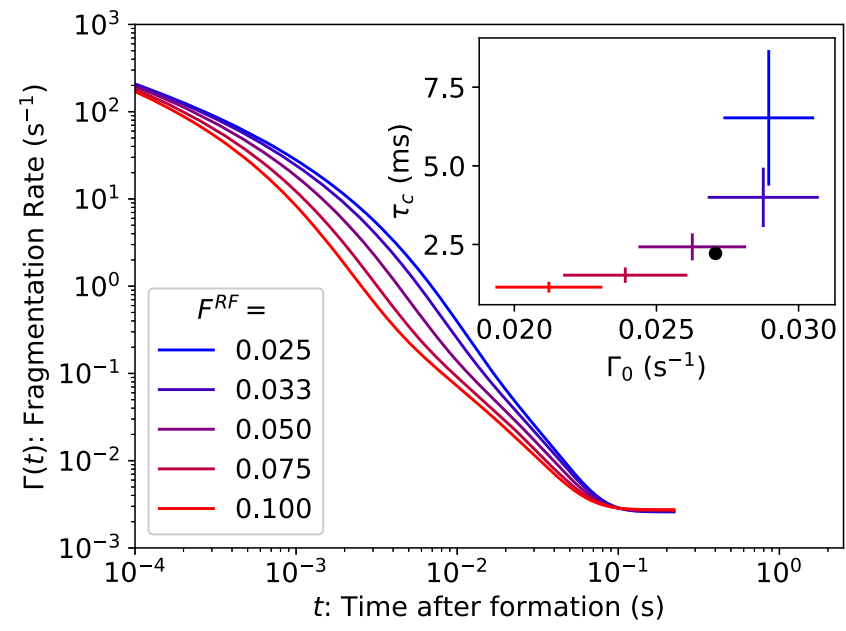

FIG. 7. Comparison of simulated spontaneous fragmentation rates $\Gamma(t)$ for different RF oscillator strengths $f^{R F}$. The inset shows fit parameters for using Eq. (7), with the black point giving the parameters of the fit to the experimental data (dashed line in Fig. 6).

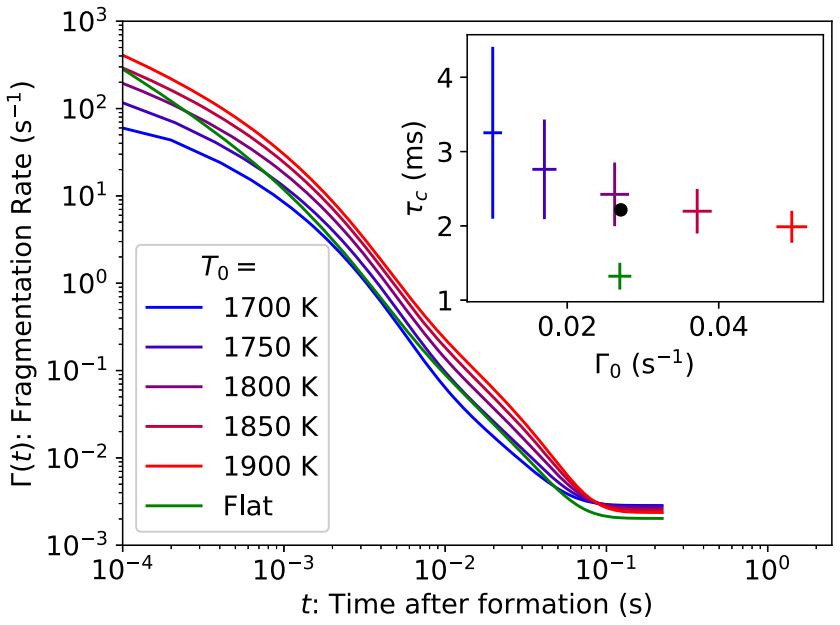

FIG. 8. Comparison of simulated spontaneous fragmentation rates $\Gamma(t)$ for different initial temperatures $T_{0}$. The inset shows fit parameters for using $\mathrm{Eq}$. (7), with the black point giving the parameters of the fit to the experimental data (dashed line in Fig. 6).

$T_{0}=1800 \mathrm{~K}$ and the storage probability of $25 \%$ constant. Unsurprisingly, the choice of $f^{R F}$ has a strong influence on the critical time $\tau_{c}$ after which RF becomes important. Fitted values for $\tau_{c}$ and $\Gamma_{0}$ according to Eq. (7) are shown in the inset of Fig. 7. From these data, the value of $f^{R F}$ that most closely reproduces the experimental decay curve is $0.055 \pm 0.011$, which is consistent with the value of 0.05 used in most of the simulations presented here.

In contrast, the choice of the temperature $T_{0}$ characterizing the initial energy distribution has a strong impact on the absolute scale of the decay rate and somewhat more subtly on the shape of the decay curve, as parameterized by $\Gamma_{0}$ and $\tau_{c}$, respectively. Figure 8 shows several simulations for different values of $T_{0}$, holding $f^{R F}$ and the storage probability constant. Also shown in Fig. 8 is a simulation for an initially flat energy distribution, i.e., $g(E, t=0)=c$, a constant. The inset shows the fitted values of $\Gamma_{0}$ and $\tau_{c}$, with the experimental values given by the black dot (error bars smaller than the symbol). The flat distribution and the $T_{0}=1800 \mathrm{~K}$ distribution give the best agreement with $\Gamma_{0}$, and the $T_{0}=1800 \mathrm{~K}$ simulation is consistent with the experimental $\tau_{c}$.

Finally, we note that it is possible to achieve similarly good agreement between the simulated and experimental decay rates while assuming $100 \%$ storage of the $\mathrm{C}_{20} \mathrm{H}_{11}{ }^{+}$daughter ions. However, this requires adjustments to the decay rate constants (e.g., increasing $\hat{k}_{\text {tot }}^{I R}$ by a factor of 3 or $\hat{k}_{2 H}^{\text {diss }}$ by a factor of 50 ), which we consider not meaningful.

\section{Cooling rate}

Beyond reproducing the measured decay rate, our master equation simulations provide insight into the time evolution of the internal energy distribution $g(E, t)$. The neutral yield $\Gamma(t)$ depends on the high-energy edge of the distribution $E_{\max }(t)$, defined in Sec. III B. The value of $E_{\max }(t)$ is plotted in Fig. 9 for the same simulation leading to the result in Fig. 6. 


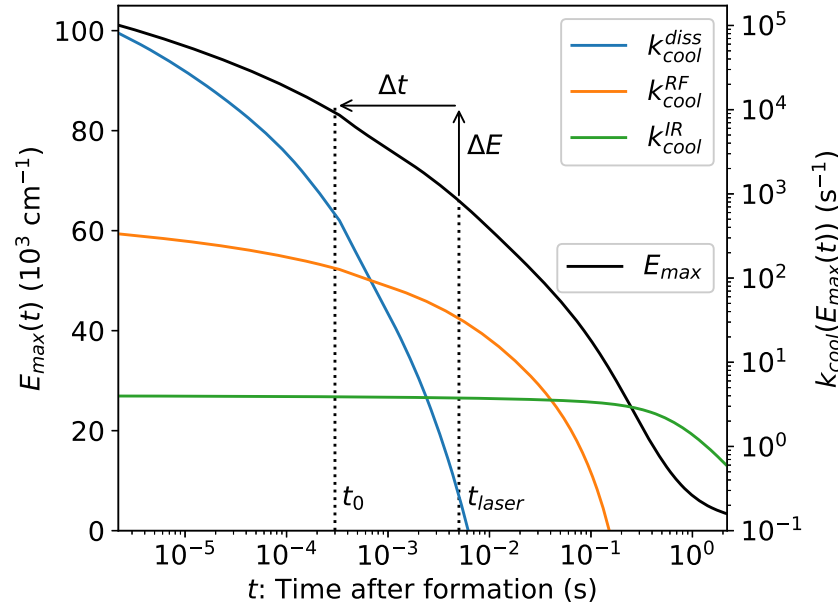

FIG. 9. Left axis: energy $E_{\max }(t)$ of the high-energy edge of the energy distribution $g(E, t)$ from master equation simulation. Right axis: ensemble cooling rates $k_{\text {cool }}=\frac{1}{E} \frac{d E}{d t}$ for modeled processes evaluated at $E_{\max }(t)$. In the laser excitation experiments, a photon absorbed at $t_{\text {laser }}$ increases $E_{\max }$ by $\Delta E$, which is equivalent to a shift in time by $\Delta t$ to the time $t_{0}$. The value of $t_{0}$ determines the laser-induced decay rate $R_{e}$.

The mean cooling rate for the ensemble as a whole is $k_{\text {cool }}$ $\equiv \frac{1}{E} \frac{d E}{d t}$. For the radiative processes, $\frac{d E}{d t}=\sum_{i} h v_{i} k_{i}^{R F / I R}(E)$, while for dissociation $\frac{d E}{d t}=\sum_{i} E k_{i}^{d i s s}(E)$. In Fig. 9, we have plotted the values of $k_{\text {cool }}$ for the modeled processes evaluated at $E_{\text {max }}(t)$. This provides a sense of the relative importance of each process as the energy distribution evolves. Dissociation is the primary cooling process (depletion cooling) during the first millisecond following formation of the ensemble. Recurrent fluorescence then dominates until about $50 \mathrm{~ms}$, after which infrared cooling becomes important.

\section{B. Laser-induced decay}

We now turn to the neutral fragment signal induced by laser excitation of the stored ion beam. We analyze the laser-induced signal following the procedure described by Sundén et al. ${ }^{49} \mathrm{Key}$ assumptions in the analysis are that photo-absorption translates the energy distribution of the ions by an energy $\Delta E$ and that this is equivalent to a translation in time by some $\Delta t$, i.e., $g\left(E+\Delta E, t_{\text {laser }}\right)$ $\propto g\left(E, t_{\text {laser }}-\Delta t\right)$, where $t_{\text {laser }}$ is the laser firing time (see Fig. 9). This approximation implies that the laser-induced neutral yield will follow the time dependence of the spontaneous decay, i.e., $R_{e}\left(t_{\text {after }}\right)$ $\propto R\left(t_{\text {laser }}-\Delta t\right)$, where $t_{\text {after }}$ is the time elapsed since the laser firing time $t_{\text {laser }}$.

Figure 10 shows three examples of the procedure used to determine $\Delta t$. The laser was tuned to the $D_{5} \leftarrow D_{0}$ transition of $\mathrm{C}_{20} \mathrm{H}_{12}{ }^{+}$ at $535 \mathrm{~nm}\left(h v=18700 \mathrm{~cm}^{-1}\right)$. The points are the inverse of the integrated laser-induced neutral counts per turn, and the solid lines are fits to the functional form,

$$
R_{e}\left(t_{\text {after }}\right)^{-1} \propto\left(t_{\text {after }}+t_{0}\right) e^{\left(t_{\text {affer }}+t_{0}\right) / \tau_{c}},
$$

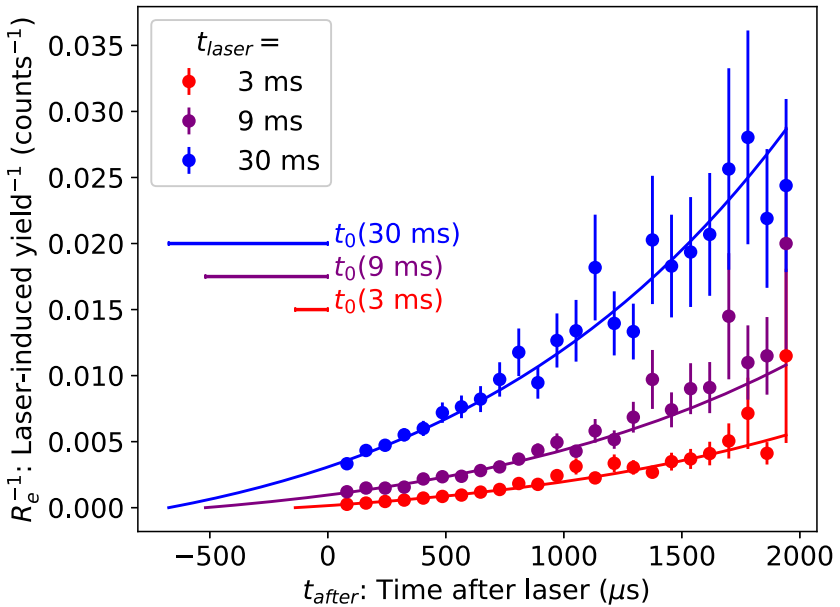

FIG. 10. Examples of the fitting procedure to determine the back-shifted time $t_{0}$. The laser-induced yield $R_{e}$ for each laser firing time $t_{\text {laser }}$ is fit to Eq. (9).

where $t_{0}=t_{\text {laser }}-\Delta t$ and $\tau_{c}$ is fixed to the value determined from the spontaneous decay measurement $(2.2 \mathrm{~ms}$, see Sec. IV A). This approach becomes uninformative as the back-shifted time $t_{0}$ approaches the critical time $\tau_{c}$ for radiation dominance and Eq. (9) becomes indistinguishable from a pure exponential, which does not have a well defined start time $t=0$.

Figure 11 plots the fitted values of $t_{0}^{-1}$ for laser firing times $t_{\text {laser }}$ from $\approx 2 \mathrm{~ms}$ to $200 \mathrm{~ms}$. Laser firing times $t_{\text {laser }} \lesssim 2 \mathrm{~ms}$ back-shift the energy distribution to times $t_{0}<0$ and are not well-represented by Eq. (9). The points on this plot trace contours of constant $\Delta E$, the absorbed photon energy. Firing times $2 \mathrm{~ms} \lesssim t_{\text {laser }} \lesssim 20 \mathrm{~ms}$ are connected to back-shifted times $100 \mu \mathrm{s} \lesssim t_{0} \lesssim 1 \mathrm{~ms}$, i.e., from times close

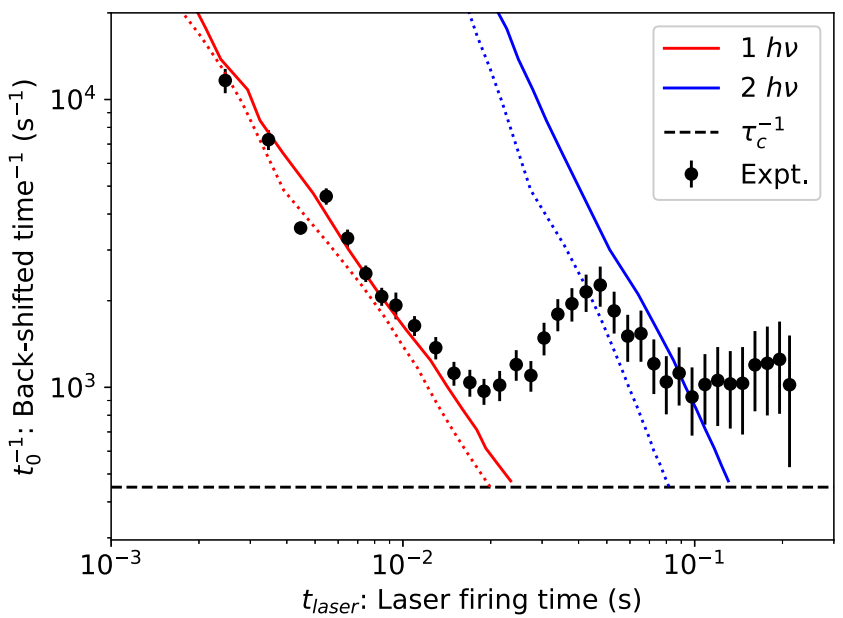

FIG. 11. Fitted values of $t_{0}^{-1}$ (black points), which trace contours of constant energy loss $\Delta E$ between the times $t_{0}$ and $t_{\text {laser. }}$. The colored lines are contours from our simulation of constant $\Delta E_{\max }=n \times h v$, where $h v=18700 \mathrm{~cm}^{-1}$ is the laser photon energy. Dotted lines are simulation results when $k_{\text {tot }}^{I R}$ is increased by a factor of two. 
to when the original source-heated ions were just entering the ring until times when radiative processes dominate the cooling. For firing times $20 \mathrm{~ms} \lesssim t_{\text {laser }} \lesssim 100 \mathrm{~ms}$, the fitted values of $t_{0}$ swing back toward earlier times before again proceeding toward the radiative limit $\tau_{c}$. This indicates that multiple photon absorption contributes to $R_{e}$.

The solid lines in Fig. 11 are from our simulations and are contours of constant $\Delta E_{\max }=n \times h v(n=1,2)$, where $E_{\max }$ is the highenergy edge of the energy distribution (see Sec. III B). The agreement with the experimental points is very good from $2 \mathrm{~ms}$ to $20 \mathrm{~ms}(n=1)$ and from $50 \mathrm{~ms}$ to $100 \mathrm{~ms}(n=2)$. The dotted lines in Fig. 11 are the same contours but for simulations where the vibrational cooling rate $k_{t o t}^{I R}$ has been increased by a factor of two. This change has little effect on the $n=1$ contour (or the spontaneous decay rates, not shown), which spans the epoch where recurrent fluorescence is the dominant cooling mechanism (see Fig. 9). The $n=2$ contour is more sensitive as vibrational cooling has begun to become relevant for laser firing times $t_{\text {laser }}>50 \mathrm{~ms}$. These results indicate that the vibrational cooling rate model is accurate to within a factor of two, which is consistent with the accuracy seen in other applications. ${ }^{29,30}$

The data in Fig. 11 show that our measured $R_{e}$ and simulated $E_{\text {max }}$ are consistent with the energy-time equivalence assumed in Eq. (9). This consistency helps us to interpret the total laser-induced yield $\Sigma_{e}=\int \Gamma_{e} d t_{\text {after }}$, where the neutral signal is integrated over the first $2 \mathrm{~ms}$ (24 turns in the storage ring) after each laser firing event. $\Gamma_{e}$ has the same relation to $R_{e}$ as given by Eq. (8), except the number of ions stored $N_{\text {store }}$ is further multiplied by another factor of $L / C$, as only the ions in the overlap section may absorb photons, and by the probability of absorbing at least one photon $(1-\mathcal{P}(0))$. The probability of absorbing $n$ photons is assumed to follow Poisson statistics,

$$
\mathcal{P}(n)=\frac{p^{n} e^{-p}}{n !}
$$

The occurrence rate of photon absorption is given by $p=J \sigma_{a b s} F / h v$ where $\sigma_{a b s}$ is the photo-absorption cross section, $F$ is the laser fluence, and $J$ is a form factor describing the overlap of the ion and laser beams. We assume that $\sigma_{a b s}$ does not change as the ions cool.

Figure 12 shows the experimental laser-induced decay rate $\Sigma_{e}$. Also shown is a comparison to our simulation, where we have included contributions of up to $n=3$ absorbed photons. For the simulated curves, we find the back-shifted time $t_{0}$ for each laser firing time using the $\Delta E_{\max }=n \times h v$ contours introduced above. We then numerically integrate a quenched power law [Eq. (7)] from $t_{0}$ to $t_{0}+$ $2 \mathrm{~ms}$ using parameters from a fit to the simulated spontaneous decay rate (Fig. 6), and weighted by the probability $\mathcal{P}(n)$. Back-shifted times $t_{0}<0$ are assumed to give an integrated yield equal to that for $t_{0}=0$. To facilitate comparison between experiment and simulation, a constant term has been added to the sum of the simulated curves, which is equal to the asymptotic yield observed in the experiment. This asymptotic yield may be due to fragmentation channels not included in the simulation or to excitation of ions which have been "reheated" through irradiation by an earlier laser shot.

The excellent agreement between the experimental and simulated $\Sigma_{e}$ curves presented in Fig. 12 was obtained assuming the photon occurrence rate $p=0.18$. For our estimated laser fluence in the laser-ion overlap region of $100 \mu \mathrm{J} / \mathrm{cm}^{2}$ and assuming perfect

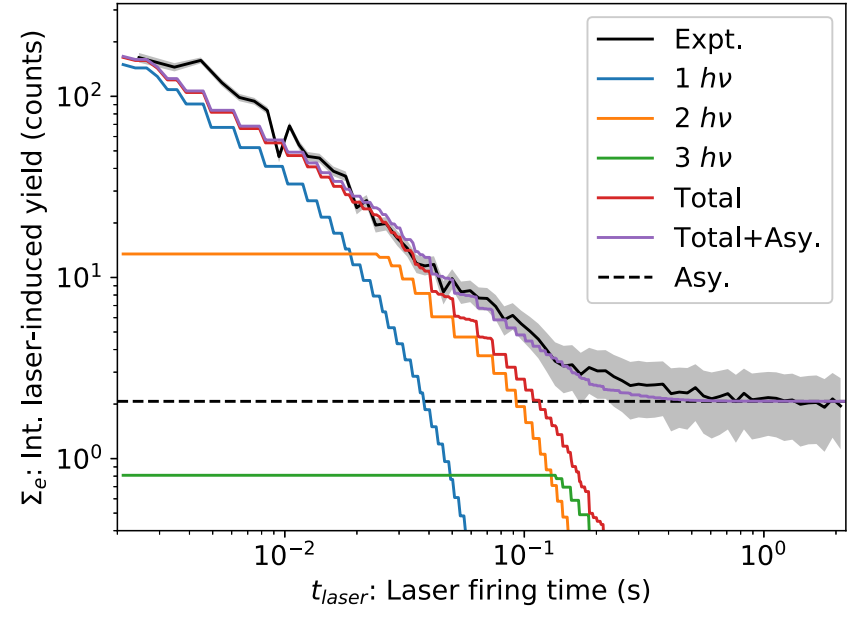

FIG. 12. Integrated laser-induced neutral yield $\Sigma_{e}=\int \Gamma_{e} d t_{\text {after. }}$. The black curve is the experimental measurement with the gray area giving the uncertainty due to counting statistics. Colored curves are based on the results of our master equation simulation and show contributions to $\Sigma_{e}$ from absorption of $n=1,2$, and 3 photons. For the sake of comparison, a constant "Asy."-equal to the asymptotic yield observed in the experiment-is added to the total simulated yield.

overlap $(J=1)$, we obtain a lower limit of $\sigma_{a b s}>670 \mathrm{Mb}$. Calculated absolute cross sections for $\mathrm{C}_{20} \mathrm{H}_{12}{ }^{+}$have been reported by Malloci et al. to reach only $60 \mathrm{Mb}$ for the $535 \mathrm{~nm}$ transition, ${ }^{50}$ a full order of magnitude below our lower limit. These calculations, however, also predict a rather broad absorption full-width at half-maximum (FWHM) of $0.43 \mathrm{eV}(100 \mathrm{~nm})$. Assuming an oscillator strength of 0.6 , which is on the high end of the range predicted by published calculations, ${ }^{45-47,51}$ our $p$ puts an upper limit on the intrinsic FWHM of $0.06 \mathrm{eV}(15 \mathrm{~nm})$. This is comparable to the FWHM of $0.09 \mathrm{eV}$ $(20 \mathrm{~nm})$ measured in a Ne matrix, ${ }^{22}$ which is presumably broadened by vibronic excitations.

\section{CONCLUSIONS}

The spontaneous and laser-induced unimolecular fragmentation of isolated perylene cations has been measured in a cryogenic electrostatic ion beam storage ring. The observed neutral yields are well-reproduced by master equation simulations, which include unimolecular dissociation of parent and co-stored daughter ions and radiative cooling by both recurrent fluorescence and vibrational infrared emission. Analysis of the time dependence of the laserinduced neutral yield shows that the vibrational cooling rate is consistent (to within a factor of two) with that predicted by the simple harmonic cascade approximation.

Our simulations allow us to extract quantitative information about the electronic transitions of $\mathrm{C}_{20} \mathrm{H}_{12}{ }^{+}$. Our spontaneous decay measurement is consistent with an emission oscillator strength for the $D_{1} \rightarrow D_{0}$ emissive transition of $f^{R F}=0.055 \pm 0.011$. Our laserinduced decay measurement sets a lower limit on the absorption cross section at $535 \mathrm{~nm}$, the center of the strong $D_{5} \leftarrow D_{0}$ excitation transition, of $\sigma_{a b s}>670 \mathrm{Mb}$. These values may serve as benchmarks for future calculations. 
Our results are consistent with the previously measured unimolecular dissociation rate constants reported by West et al. ${ }^{37}$ Without these experimentally benchmarked absolute rate constants, the pre-exponential factors $A_{R}^{\text {diss }}$ could be treated as adjustable parameters. Alternatively, time-dependent action spectroscopy could be employed to track cooling rates without reliance on absolute dissociation rates.

Our methodology could be further improved by explicitly accounting for the kinetic energy release (KER) lost during dissociation. Measurement and modeling of the KER distribution could help constrain the storage probability $\epsilon_{\text {store }}$ of the $\mathrm{C}_{20} \mathrm{H}_{11}{ }^{+}$daughter ions, which is here adjusted ad hoc to obtain agreement between experiment and simulation. Variation of KER with internal energy might also lead to a time-dependent detection efficiency.

Quantitative models of the unimolecular dissociation and radiative cooling of PAH ions are of key importance in astrochemistry. Experimental tests, such as those presented in this article for perylene, contribute toward refining these models.

\section{SUPPLEMENTARY MATERIAL}

See the supplementary material for complete tabulation of harmonic frequencies and IR intensities, and a tabulation of the rate constants used in the master equation simulation.

\section{ACKNOWLEDGMENTS}

The authors thank Brandi West for helpful discussions. This work was supported by the Swedish Research Council (Grant Nos. 2016-03675, 2016-04181, and 2018-04092), the Carl Trygger Foundation (Grant No. 17:436), and the Swedish Foundation for International Collaboration in Research and Higher Education (STINT, Grant No. PT2017-7328 awarded to J.N.B., E.C., and M.H.S.). We acknowledge the DESIREE infrastructure for provisioning of facilities and experimental support and thank the operators and technical staff for their invaluable assistance. The DESIREE infrastructure receives funding from the Swedish Research Council under the Grant No. 2017-00621. This article is based upon the work from COST Action CA18212-Molecular Dynamics in the GAS phase (MD-GAS), supported by COST (European Cooperation in Science and Technology). M.H.S, H.T.S., and H.Z. acknowledge support from the project grant "Probing charge- and mass- transfer reactions on the atomic level" (2018.0028) from the Knut and Alice Wallenberg Foundation.

\section{DATA AVAILABILITY}

The data that support the findings of this study are available from the corresponding author upon reasonable request.

\section{REFERENCES}

${ }^{1}$ A. G. G. M. Tielens, Annu. Rev. Astron. Astrophys. 46, 289 (2008).

${ }^{2}$ A. G. G. M. Tielens, Rev. Mod. Phys. 85, 1021 (2013).

${ }^{3}$ V. Mennella, L. Hornekær, J. Thrower, and M. Accolla, Astrophys. J. Lett. 745, L2 (2012).

${ }^{4}$ J. D. Thrower, B. Jørgensen, E. E. Friis, S. Baouche, V. Mennella, A. C. Luntz, M. Andersen, B. Hammer, and L. Hornekær, Astrophys. J. 752, 3 (2012).
${ }^{5}$ G. Reitsma, L. Boschman, M. J. Deuzeman, O. González-Magaña, S. Hoekstra, S. Cazaux, R. Hoekstra, and T. Schlathölter, Phys. Rev. Lett. 113, 053002 (2014).

${ }^{6}$ M. Gatchell, M. H. Stockett, N. de Ruette, T. Chen, L. Giacomozzi, R. F. Nascimento, M. Wolf, E. K. Anderson, R. Delaunay, V. Vizcaino, P. Rousseau, L. Adoui, B. A. Huber, H. T. Schmidt, H. Zettergren, and H. Cederquist, Phys. Rev. A 92, 050702(R) (2015)

${ }^{7}$ M. H. Stockett, M. Gatchell, T. Chen, N. de Ruette, L. Giacomozzi, M. Wolf, H. T. Schmidt, H. Zettergren, and H. Cederquist, J. Phys. Chem. Lett. 6, 4504-4509 (2015).

${ }^{8}$ E. R. Micelotta, A. P. Jones, and A. G. G. M. Tielens, Astron. Astrophys. 510, A36 (2010); arXiv:0910.2461 [astro-ph.GA].

${ }^{9}$ L. J. Allamandola, A. G. G. M. Tielens, and J. R. Barker, Astrophys. J., Suppl. Ser. 71, 733 (1989).

${ }^{10}$ S. Martin, J. Bernard, R. Brédy, B. Concina, C. Joblin, M. Ji, C. Ortega, and L. Chen, Phys. Rev. Lett. 110, 063003 (2013).

${ }^{11}$ S. Martin, M. Ji, J. Bernard, R. Brédy, B. Concina, A. R. Allouche, C. Joblin, C. Ortega, G. Montagne, A. Cassimi, Y. Ngono-Ravache, and L. Chen, Phys. Rev. A 92, 053425 (2015).

${ }^{12}$ V. Chandrasekaran, B. Kafle, A. Prabhakaran, O. Heber, M. Rappaport, H. Rubinstein, D. Schwalm, Y. Toker, and D. Zajfman, J. Phys. Chem. Lett. 5, 4078 (2014).

${ }^{13}$ G. Ito, T. Furukawa, H. Tanuma, J. Matsumoto, H. Shiromaru, T. Majima, M. Goto, T. Azuma, and K. Hansen, Phys. Rev. Lett. 112, 183001 (2014).

${ }^{14}$ A. Léger, P. Boissel, and L. d'Hendecourt, Phys. Rev. Lett. 60, 921 (1988).

${ }^{15}$ M. Saito, H. Kubota, K. Yamasa, K. Suzuki, T. Majima, and H. Tsuchida, Phys. Rev. A 102, 012820 (2020).

${ }^{16}$ J. Bernard, L. Chen, R. Brédy, M. Ji, C. Ortéga, J. Matsumoto, and S. Martin, in Proceedings of the 18th International Conference on the Physics of Highly Charged Ions (HCI-2016), Kielce, Poland, 11-16 September 2016 [Nucl. Instrum. Methods Phys. Res., Sec. B 408, 21 (2017)].

${ }^{17}$ M. Ji, J. Bernard, L. Chen, R. Brédy, C. Ortéga, C. Joblin, A. Cassimi, and S. Martin, J. Chem. Phys. 146, 044301 (2017).

${ }^{18}$ J. Bernard, A. Al-Mogeeth, A.-R. Allouche, L. Chen, G. Montagne, and S. Martin, J. Chem. Phys. 150, 054303 (2019).

${ }^{19}$ M. H. Stockett, M. Björkhage, H. Cederquist, H. T. Schmidt, and H. Zettergren, Faraday Discuss. 217, 126-137 (2019).

${ }^{20}$ M. Wolf, H. V. Kiefer, J. Langeland, L. H. Andersen, H. Zettergren, H. T. Schmidt, H. Cederquist, and M. H. Stockett, Astrophys. J. 832, 24 (2016).

${ }^{21}$ C. Joblin, F. Salama, and L. Allamandola, J. Chem. Phys. 102, 9743 (1995).

${ }^{22}$ X. D. F. Chillier, B. M. Stone, C. Joblin, F. Salama, and L. J. Allamandola, J. Chem. Phys. 116, 5725 (2002).

${ }^{23}$ R. D. Thomas, H. T. Schmidt, G. Andler, M. Björkhage, M. Blom, L. Brännholm, E. Bäckström, H. Danared, S. Das, N. Haag, P. Halldén, F. Hellberg, A. I. S. Holm, H. A. B. Johansson, A. Källberg, G. Källersjö, M. Larsson, S. Leontein, L. Liljeby, P. Löfgren, B. Malm, S. Mannervik, M. Masuda, D. Misra, A. Orbán, A. Paál, P. Reinhed, K.-G. Rensfelt, S. Rosén, K. Schmidt, F. Seitz, A. Simonsson, J. Weimer, H. Zettergren, and H. Cederquist, Rev. Sci. Instrum. 82, 065112 (2011).

${ }^{24}$ H. T. Schmidt, R. D. Thomas, M. Gatchell, S. Rosén, P. Reinhed, P. Löfgren, L. Brännholm, M. Blom, M. Björkhage, E. Bäckström, J. D. Alexander, S. Leontein, D. Hanstorp, H. Zettergren, L. Liljeby, A. Källberg, A. Simonsson, F. Hellberg, S. Mannervik, M. Larsson, W. D. Geppert, K. G. Rensfelt, H. Danared, A. Paál, M. Masuda, P. Halldén, G. Andler, M. H. Stockett, T. Chen, G. Källersjö, J. Weimer, K. Hansen, H. Hartman, and H. Cederquist, Rev. Sci. Instrum. 84, 055115 (2013).

${ }^{25}$ E. Bäckström, D. Hanstorp, O. M. Hole, M. Kaminska, R. F. Nascimento, M. Blom, M. Björkhage, A. Källberg, P. Löfgren, P. Reinhed, S. Rosén, A. Simonsson, R. D. Thomas, S. Mannervik, H. T. Schmidt, and H. Cederquist, Phys. Rev. Lett. 114, 143003 (2015).

${ }^{26}$ A. P. O’Connor, A. Becker, K. Blaum, C. Breitenfeldt, S. George, J. Göck, M. Grieser, F. Grussie, E. A. Guerin, R. von Hahn, U. Hechtfischer, P. Herwig, J. Karthein, C. Krantz, H. Kreckel, S. Lohmann, C. Meyer, P. M. Mishra, O. Novotný, R. Repnow, S. Saurabh, D. Schwalm, K. Spruck, S. Sunil Kumar, S. Vogel, and A. Wolf, Phys. Rev. Lett. 116, 113002 (2016). 
${ }^{27}$ C. Meyer, A. Becker, K. Blaum, C. Breitenfeldt, S. George, J. Göck, M. Grieser, F. Grussie, E. A. Guerin, R. von Hahn, P. Herwig, C. Krantz, H. Kreckel, J. Lion, S. Lohmann, P. M. Mishra, O. Novotný, A. P. O'Connor, R. Repnow, S. Saurabh, D. Schwalm, L. Schweikhard, K. Spruck, S. Sunil Kumar, S. Vogel, and A. Wolf, Phys. Rev. Lett. 119, 023202 (2017).

${ }^{28}$ C. Breitenfeldt, K. Blaum, S. George, J. Göck, G. Guzmán-Ramírez, J. Karthein, T. Kolling, M. Lange, S. Menk, C. Meyer, J. Mohrbach, G. Niedner-Schatteburg, D. Schwalm, L. Schweikhard, and A. Wolf, Phys. Rev. Lett. 120, 253001 (2018).

${ }^{29}$ J. N. Bull, M. S. Scholz, E. Carrascosa, M. K. Kristiansson, G. Eklund, N. Punnakayathil, N. de Ruette, H. Zettergren, H. T. Schmidt, H. Cederquist, and M. H. Stockett, J. Chem. Phys. 151, 114304 (2019).

${ }^{30}$ M. H. Stockett, J. N. Bull, J. T. Buntine, E. Carrascosa, E. K. Anderson, M. Gatchell, M. Kaminska, R. F. Nascimento, H. Cederquist, H. T. Schmidt, and H. Zettergren, Eur. Phys. J. D 74, 150 (2020).

${ }^{31}$ E. K. Anderson, "DESIREE: Instrumentation developments and hot metal cluster decays," Ph.D. thesis, Department of Physics, Stockholm University, 2019.

${ }^{32}$ T. Beyer and D. F. Swinehart, Commun. ACM 16, 379 (1973).

${ }^{33}$ J.-D. Chai and M. Head-Gordon, Phys. Chem. Chem. Phys. 10, 6615 (2008).

${ }^{34}$ T. H. Dunning, Jr., J. Chem. Phys. 90, 1007 (1989).

${ }^{35}$ M. J. Frisch, G. W. Trucks, H. B. Schlegel, G. E. Scuseria, M. A. Robb, J. R. Cheeseman, G. Scalmani, V. Barone, B. Mennucci, G. A. Petersson, H. Nakatsuji, M. Caricato, X. Li, H. P. Hratchian, A. F. Izmaylov, J. Bloino, G. Zheng, J. L. Sonnenberg, M. Hada, M. Ehara, K. Toyota, R. Fukuda, J. Hasegawa, M. Ishida, T. Nakajima, Y. Honda, O. Kitao, H. Nakai, T. Vreven, J. A. Montgomery, Jr., J. E. Peralta, F. Ogliaro, M. Bearpark, J. J. Heyd, E. Brothers, K. N. Kudin, V. N. Staroverov, R. Kobayashi, J. Normand, K. Raghavachari, A. Rendell, J. C. Burant, S. S. Iyengar, J. Tomasi, M. Cossi, N. Rega, J. M. Millam, M. Klene, J. E. Knox, J. B. Cross, V. Bakken, C. Adamo, J. Jaramillo, R. Gomperts, R. E. Stratmann, O. Yazyev, A. J. Austin, R. Cammi, C. Pomelli, J. W. Ochterski, R. L. Martin, K. Morokuma, V. G. Zakrzewski, G. A. Voth, P. Salvador, J. J. Dannenberg, S. Dapprich, A. D. Daniels, C. Farkas, J. B. Foresman, J. V. Ortiz,
J. Cioslowski, and D. J. Fox, Gaussian 16, Revision B.01, Gaussian, Inc., Wallingford, CT, 2016.

${ }^{36}$ P. Boissel, P. de Parseval, P. Marty, and G. Lefèvre, J. Chem. Phys. 106, 4973 (1997).

${ }^{37}$ B. West, S. Rodriguez Castillo, A. Sit, S. Mohamad, B. Lowe, C. Joblin, A. Bodi, and P. M. Mayer, Phys. Chem. Chem. Phys. 20, 7195 (2018).

${ }^{38}$ W. Forst, J. Phys. Chem. 76, 342 (1972).

${ }^{39}$ W. D. Price, P. D. Schnier, and E. R. Williams, J. Phys. Chem. B 101, 664 (1997).

${ }^{40}$ K. Hansen, M. H. Stockett, M. Kaminska, R. F. Nascimento, E. K. Anderson, M. Gatchell, K. C. Chartkunchand, G. Eklund, H. Zettergren, H. T. Schmidt, and H. Cederquist, Phys. Rev. A 95, 022511 (2017).

${ }^{41}$ K. Hansen, J. U. Andersen, P. Hvelplund, S. P. Møller, U. V. Pedersen, and V. V. Petrunin, Phys. Rev. Lett. 87, 123401 (2001).

${ }^{42}$ M. Barat, J. C. Brenot, J. A. Fayeton, and Y. J. Picard, Rev. Sci. Instrum. 71, 2050 (2000).

${ }^{43}$ M. Krems, J. Zirbel, M. Thomason, and R. D. DuBois, Rev. Sci. Instrum. 76, 093305 (2005).

${ }^{44}$ V. K. Jain and Z. H. Zaidi, Spectrochim. Acta, A 43, 1275 (1987).

${ }^{45}$ F. Negri and M. Z. Zgierski, J. Chem. Phys. 100, 1387 (1994).

${ }^{46}$ T. M. Halasinski, J. L. Weisman, R. Ruiterkamp, T. J. Lee, F. Salama, and M. Head-Gordon, J. Phys. Chem. A 107, 3660 (2003).

${ }^{47}$ S. Hirata, M. Head-Gordon, J. Szczepanski, and M. Vala, J. Phys. Chem. A 107, 4940 (2003).

${ }^{48}$ A. M. Tokmachev, M. Boggio-Pasqua, D. Mendive-Tapia, M. J. Bearpark, and M. A. Robb, J. Chem. Phys. 132, 044306 (2010).

${ }^{49}$ A. E. K. Sundén, M. Goto, J. Matsumoto, H. Shiromaru, H. Tanuma, T. Azuma, J. U. Andersen, S. E. Canton, and K. Hansen, Phys. Rev. Lett. 103, 143001 (2009).

${ }^{50}$ G. Malloci, G. Mulas, and C. Joblin, Astron. Astrophys. 426, 105 (2004).

${ }^{51}$ S. Hirata, T. J. Lee, and M. Head-Gordon, J. Chem. Phys. 111, 8904 (1999). 